\title{
DIRECTED POLYMER IN RANDOM ENVIRONMENT AND LAST PASSAGE PERCOLATION*
}

\author{
Philippe CARMOnA ${ }^{1}$
}

\begin{abstract}
The sequence of random probability measures $\nu_{n}$ that gives a path of length $n, \frac{1}{n}$ times the sum of the random weights collected along the paths, is shown to satisfy a large deviations principle with good rate function the Legendre transform of the free energy of the associated directed polymer in a random environment. Consequences on the asymptotics of the typical number of paths whose collected weight is above a fixed proportion are then drawn.
\end{abstract}

Mathematics Subject Classification. 60K37.

Received June 12, 2008.

\section{INTRODUCTION}

\section{Last passage percolation}

To each site $(k, x)$ of $\mathbb{N} \times \mathbb{Z}^{d}$ is assigned a random weight $\eta(k, x)$. The $(\eta(k, x))_{k \geq 1, x \in \mathbb{Z}^{d}}$ are taken IID under the probability measure $\mathbf{Q}$.

The set of oriented paths of length $n$ starting from the origin is

$$
\Omega_{n}=\left\{\omega=\left(\omega_{0}, \ldots, \omega_{n}\right): \omega_{i} \in \mathbb{Z}^{d}, \omega_{0}=0,\left|\omega_{i}-\omega_{i-1}\right|=1\right\} .
$$

The weight (energy, reward) of a path is the sum of weights of visited sites:

$$
H_{n}=H_{n}(\omega, \eta)=\sum_{k=1}^{n} \eta\left(k, \omega_{k}\right) \quad\left(n \geq 1, \omega \in \Omega_{n}\right) .
$$

Observe that when $\eta(k, x)$ are $\operatorname{Bernoulli}(p)$ distributed

$$
\mathbf{Q}(\eta(k, x)=1)=1-\mathbf{Q}(\eta(k, x)=0)=p \in(0,1),
$$

the quantity $\frac{H_{n}}{n}(\omega, \eta)$ is the proportion of open sites visited by $\omega$, and it is natural to consider for $p<\rho<1$,

$$
N_{n}(\rho)=\text { number of paths of length } n \text { such that } H_{n}(\omega, \eta) \geq n \rho .
$$

Keywords and phrases. Directed polymer, random environment, partition function, last passage percolation.

* The author acknowledges the support of the French Ministry of Education through the ANR BLAN07-2184264 grant.

1 Laboratoire Jean Leray, UMR 6629 Université de Nantes, BP 92208, 44322 Nantes Cedex 03, France;

http://www.math.sciences.univ-nantes.fr/〜 carmona; philippe.carmona@math.univ-nantes.fr 
The problem of $\rho$-percolation, as we learnt it from Comets et al. [9] and Kesten and Sidoravicius [12], is to study the behaviour of $N_{n}(\rho)$ for large $n$.

\section{Directed polymer in a random environment}

We are going to consider fairly general environment distributions, by requiring first that they have exponential moments of any order:

$$
\lambda(\beta)=\log \mathbf{Q}\left(\mathrm{e}^{\beta \eta(k, x)}\right)<+\infty \quad(\beta \in \mathbb{R}),
$$

and second that they satisfy a logarithmic Sobolev inequality (see e.g. [2]): in particular we can apply our result to bounded support and Gaussian environments.

The polymer measure is the random probability measure defined on the set of oriented paths of length $n$ by:

$$
\mu_{n}(\omega)=(2 d)^{-n} \frac{\mathrm{e}^{\beta H_{n}(\omega, \eta)}}{Z_{n}(\beta)} \quad\left(\omega \in \Omega_{n}\right),
$$

with $Z_{n}(\beta)$ the partition function

$$
Z_{n}(\beta)=Z_{n}(\beta, \eta)=(2 d)^{-n} \sum_{\omega \in \Omega_{n}} \mathrm{e}^{\beta H_{n}(\omega, \eta)}=\mathbf{P}\left(\mathrm{e}^{\beta H_{n}(\omega, \eta)}\right)
$$

where $\mathbf{P}$ is the law of simple random walk on $\mathbb{Z}^{d}$ starting from the origin.

Bolthausen [3] proved the existence of a deterministic limiting free energy

$$
p(\beta)=\lim _{n \rightarrow+\infty} \frac{1}{n} \mathbf{Q}\left(\log Z_{n}(\beta)\right)=\mathbf{Q} \text { a.s. } \lim _{n \rightarrow+\infty} \frac{1}{n} \log Z_{n}(\beta) .
$$

Thanks to Jensen's inequality, we have the upper bound $p(\beta) \leq \lambda(\beta)$ and it is conjectured (and partially proved, see $[6,7])$ that the behaviour of a typical path under the polymer measure is diffusive iff $\beta \in \mathcal{C}_{\eta}$ the critical region

$$
\mathcal{C}_{\eta}=\{\beta \in \mathbb{R}: p(\beta)=\lambda(\beta)\} .
$$

In dimension $d=1, \mathcal{C}_{\eta}=\{0\}$ and in dimensions $d \geq 3, \mathcal{C}_{\eta}$ contains a neighborhood of the origin (see [3,8]).

\section{The main theorem}

The connection between Last passage percolation and Directed polymer in random environment is made by the family $\left(\nu_{n}\right)_{n \in \mathbb{N}}$ of random probability measures on the real line:

$$
\nu_{n}(A)=\frac{1}{\left|\Omega_{n}\right|} \sum_{\omega \in \Omega_{n}} \mathbf{1}_{\left(\frac{H_{n}}{n}(\omega, \eta) \in A\right)}=\mathbf{P}\left(\frac{H_{n}}{n}(\omega, \eta) \in A\right) .
$$

Indeed,

$$
N_{n}(\rho)=\sum_{\omega \in \Omega_{n}} \mathbf{1}_{\left(H_{n}(\omega, \eta) \geq n \rho\right)}=(2 d)^{n} \nu_{n}([\rho,+\infty)) .
$$

The main result of the paper is

Theorem 1.1. Q almost surely, the family $\left(\nu_{n}\right)_{n \in \mathbb{N}}$ satisfies a large deviations principle with good rate function $I=p^{*}$ the Legendre transform of the free energy of the directed polymer.

Let $m=\mathbf{Q}(\eta(k, x))$ be the average weight of a path $m=\mathbf{Q}\left(\frac{H_{n}}{n}(\omega, \eta)\right)$. It is natural to consider the quantities:

$$
N_{n}(\rho)= \begin{cases}\sum_{\omega \in \Omega_{n}} \mathbf{1}_{\left(H_{n}(\omega, \eta) \geq n \rho\right)} & \text { if } \rho \geq m, \\ \sum_{\omega \in \Omega_{n}} \mathbf{1}_{\left(H_{n}(\omega, \eta) \leq n \rho\right)} & \text { if } \rho<m .\end{cases}
$$


A simple exchange of limits $\beta \rightarrow \pm \infty$, and $n \rightarrow+\infty$, yields the following

$$
\rho^{ \pm}=\mathbf{Q} \text { a.s. } \lim _{n \rightarrow+\infty} \max _{\omega \in \Omega_{n}} \pm \frac{H_{n}}{n}(\omega, \eta)=\lim _{\beta \rightarrow+\infty} \frac{p( \pm \beta)}{\beta} \in[0,+\infty] .
$$

Repeating the proof of Theorem 1.1 of [9] gives

Corollary 1.2. For $-\rho^{-}<\rho<\rho^{+}$, we have $\mathbf{Q}$ almost surely,

$$
\lim _{n \rightarrow+\infty}\left(N_{n}(\rho)\right)^{\frac{1}{n}}=(2 d) \mathrm{e}^{-I(\rho)} .
$$

We can then translate our knowledge of the critical region $\mathcal{C}_{\eta}$, into the following remark. Let

$$
\mathcal{V}_{\eta}=\left\{\rho \in \mathbb{R}: I(\rho)=\lambda^{*}(\rho)\right\} .
$$

In dimension $d=1, \mathcal{V}_{\eta}=\{m\}$ and in dimensions $d \geq 3, \mathcal{V}_{\eta}$ contains a neighbourhood of $m$.

This means that in dimensions $d \geq 3$, the typical large deviation of $\frac{H_{n}}{n}(\omega, \eta)$ close to its mean is the same as the large deviation of $\frac{1}{n}\left(\eta_{1}+\cdots+\eta_{n}\right)$ close to its mean, with $\eta_{i}$ IID. There is no influence of the path $\omega$ : this gives another justification to the name weak-disorder region given to the critical set $\mathcal{C}_{\eta}$.

\section{Proof of the MAIN TheOrem}

Observe that for any $\beta \in \mathbb{R}$ we have:

$$
\int \mathrm{e}^{\beta n x} \mathrm{~d} \nu_{n}(x)=\mathbf{P}\left(\mathrm{e}^{\beta H_{n}(\omega, \eta)}\right)=Z_{n}(\beta) \quad \mathbf{Q} \text { a.s. }
$$

Consequently, since $\mathrm{e}^{u}+\mathrm{e}^{-u} \geq \mathrm{e}^{|u|}$, we obtain for any $\beta>0$,

$$
\limsup _{n \rightarrow+\infty} \frac{1}{n} \log \left(\int \mathrm{e}^{\beta n|x|} \mathrm{d} \nu_{n}(x)\right) \leq p(\beta)+p(-\beta)<+\infty
$$

and the family $\left(\nu_{n}\right)_{n \geq 0}$ is exponentially tight (see the Appendix, Lem. A.1). We only need to show now that for a lower semicontinuous function $I$, and for $x \in \mathbb{R}$

$$
\begin{aligned}
& \lim _{\delta \rightarrow 0} \liminf _{n \rightarrow \infty} \frac{1}{n} \log \nu_{n}((x-\delta, x+\delta))=-I(x), \\
& \lim _{\delta \rightarrow 0} \limsup _{n \rightarrow \infty} \frac{1}{n} \log \nu_{n}([x-\delta, x+\delta])=-I(x) .
\end{aligned}
$$

From these, we shall infer that $\left(\nu_{n}\right)_{n \in \mathbb{N}}$ follows a large deviations principle with good rate function $I$. Eventually, equation (2.1) and

$$
\lim _{n \rightarrow \infty} \frac{1}{n} \log Z_{n}(\beta)=p(\beta)
$$

will imply, by Varadhan's lemma that $I$ and $p$ are Legendre conjugate:

$$
I(x)=p^{*}(x)=\sup _{\beta \in \mathbb{R}}(x \beta-p(\beta)) .
$$

The strategy of proof finds its origin in Varadhan's seminal paper [13], and has already successfully been applied in [5]. Let us define for $\lambda>0, x \in \mathbb{Z}, a \in \mathbb{R}$

$$
V_{n}^{(\lambda)}(x, a ; \eta)=\log \mathbf{P}^{x}\left(\mathrm{e}^{-\lambda\left|H_{n}(\omega, \eta)-a\right|}\right)=V^{(\lambda)}\left(0, a ; \tau_{o, x} \circ \eta\right),
$$


with $\tau_{k, x}$ the translation operator on the environment defined by:

$$
\tau_{k, x} \circ \eta(i, y)=\eta(k+i, x+y),
$$

and $\mathbf{P}^{x}$ the law of simple random walk starting from $x$.

Step 1 . The functions $v_{n}^{(\lambda)}(a)=\mathbf{Q}\left(V^{(\lambda)}(0, a ; \eta)\right)$ satisfy the inequality

$$
v_{n+m}^{(\lambda)}(a+b) \geq v_{n}^{(\lambda)}(a)+v_{m}^{(\lambda)}(b) \quad(n, m \in \mathbb{N} ; a, b \in \mathbb{R}) .
$$

Proof. Since $\left|H_{n+m}-(a+b)\right| \leq\left|H_{n}-b\right|+\left|\left(H_{n+m}-H_{n}\right)-a\right|$ we have

$$
\begin{aligned}
V_{n+m}^{(\lambda)}(x, a+b ; \eta) & \geq \log \mathbf{P}^{x}\left(\mathrm{e}^{-\lambda\left|H_{n}-b\right|} \mathrm{e}^{-\lambda\left|\left(H_{n+m}-H_{n}\right)-a\right|}\right) \\
& =\log \mathbf{P}^{x}\left(\mathrm{e}^{-\lambda\left|H_{n}-b\right|} \mathrm{e}^{V_{m}^{(\lambda)}\left(0, a ; \tau_{n, S_{n}} \circ \eta\right)}\right) \\
& =\log \sum_{y} \mathbf{P}^{x}\left(\mathrm{e}^{-\lambda\left|H_{n}-b\right|} \mathbf{1}_{\left(S_{n}=y\right)}\right) \mathrm{e}^{V_{m}^{(\lambda)}\left(0, a ; \tau_{n, y} \circ \eta\right)} \\
& =V_{n}^{(\lambda)}(x, b ; \eta)+\log \left(\sum_{y} \sigma_{n}(y) \mathrm{e}^{V_{m}^{(\lambda)}\left(0, a ; \tau_{n, y} \circ \eta\right)}\right) \\
& \geq V_{n}^{(\lambda)}(x, b ; \eta)+\sum_{y} \sigma_{n}(y) V_{m}^{(\lambda)}\left(0, a ; \tau_{n, y} \circ \eta\right)
\end{aligned}
$$

(Jensen's inequality),

with $\sigma_{n}$ the probability measure on $\mathbb{Z}^{d}$ :

$$
\sigma_{n}(y)=\frac{1}{V_{n}^{(\lambda)}(x, b ; \eta)} \mathbf{P}^{x}\left(\mathrm{e}^{-\lambda\left|H_{n}-b\right|} \mathbf{1}_{\left(S_{n}=y\right)}\right) \quad\left(y \in \mathbb{Z}^{d}\right) .
$$

Observe that the random variables $\sigma_{n}(y)$ are measurable with respect to the sigma field $\mathcal{G}_{n}=\sigma(\eta(i, x): i \leq$ $\left.n, x \in \mathbb{Z}^{d}\right)$, whereas the random variables $V_{m}^{(\lambda)}\left(0, a ; \tau_{n, y} \circ \eta\right)$ are independent from $\mathcal{G}_{n}$. Hence, by stationarity,

$$
\begin{aligned}
v_{n+m}^{(\lambda)}(a+b) & =\mathbf{Q}\left(V_{n+m}^{(\lambda)}(0, a+b ; \eta)\right) \\
& \geq v_{n}^{(\lambda)}(b)+\sum_{y} \mathbf{Q}\left(\sigma_{n}(y)\right) \mathbf{Q}\left(V_{m}^{(\lambda)}\left(0, a ; \tau_{n, y} \circ \eta\right)\right) \\
& =v_{n}^{(\lambda)}(b)+\sum_{y} \mathbf{Q}\left(\sigma_{n}(y)\right) v_{m}^{(\lambda)}(a) \\
& =v_{n}^{(\lambda)}(b)+v_{m}^{(\lambda)}(a) \mathbf{Q}\left(\sum_{y} \sigma_{n}(y)\right) \\
& =v_{n}^{(\lambda)}(b)+v_{m}^{(\lambda)}(a) .
\end{aligned}
$$

Step 2. There exists a function $I^{(\lambda)}: \mathbb{R} \rightarrow \mathbb{R}^{+}$convex, non negative, Lipschitz with constant $\lambda$, such that

$$
\left.-\lim _{n \rightarrow \infty} \frac{1}{n} v_{n}^{(\lambda)}\left(a_{n}\right)=I^{(\lambda)}(\xi) \quad\left(\text { if } \frac{a_{n}}{n} \rightarrow \xi \in \mathbb{R}\right)\right) .
$$

Proof. From $\left|H_{n}-a\right| \leq\left|H_{n}-b\right|+|a-b|$ we infer that

$$
V_{n}^{(\lambda)}(0, a ; \eta) \geq V_{n}^{(\lambda)}(0, b ; \eta)-\lambda|a-b|
$$


Therefore the functions $v_{n}^{(\lambda)}$ are all Lipschitz continuous with the same constant $\lambda$ and we combine this fact with standard subadditivity arguments (see e.g. Varadhan [13] or Alexander [1]). For sake of completeness, we give a detailed proof in the Appendix Lemma A.2.

Step 3. Q almost surely, for any $\xi \in \mathbb{R}$, if $\frac{a_{n}}{n} \rightarrow \xi$, then

$$
\lim _{n \rightarrow \infty}-\frac{1}{n} \log \mathbf{P}\left(\mathrm{e}^{-\lambda\left|H_{n}-a_{n}\right|}\right)=I^{(\lambda)}(\xi) .
$$

Proof. Since the functions are Lipschitz, it is enough to prove that for any fixed $\xi \in \mathbb{Q},(2.6)$ holds a.s. This is where we use the restrictive assumptions made on the distribution of the environment. If the distribution of $\eta$ is with bounded support, or Gaussian, or more generally satisfies a logarithmic Sobolev inequality with constant $c>0$, then it has the Gaussian concentration of measure property (see [2]): for any 1-Lipschitz function $F$ of independent random variables distributed as $\eta$,

$$
\mathbf{P}(|F-\mathbf{P}(F)| \geq r) \leq 2 \mathrm{e}^{-r^{2} / c} \quad(r>0)
$$

It is easy to prove, as in Proposition 1.4 of [4], that the function

$$
(\eta(k, x), k \leq n,|x| \leq n) \rightarrow \log \mathbf{P}\left(\mathrm{e}^{-\lambda\left|H_{n}(\omega, \eta)-a\right|}\right)
$$

is Lipschitz, with respect to the Euclidean norm, with Lipschitz constant at most $\lambda \sqrt{n}$. Therefore, the Gaussian concentration of measure yields

$$
\mathbf{Q}\left(\left|V_{n}^{(\lambda)}(0, a ; \eta)-v_{n}^{(\lambda)}(a)\right| \geq u\right) \leq 2 \mathrm{e}^{-\frac{\lambda^{2} u^{2}}{c n}}
$$

We conclude by a Borel Cantelli argument combined with (2.5).

Observe that for fixed $\xi \in \mathbb{R}$, the function $\lambda \rightarrow I^{(\lambda)}(\xi)$ is increasing; we shall consider the limit:

$$
I(\xi)=\lim _{\lambda \uparrow+\infty} \uparrow I^{(\lambda)}(\xi)
$$

which is by construction non negative, convex and lower semi continuous.

Step 4. The function $I$ satisfy (2.2) and (2.3).

Proof. Given, $\xi \in \mathbb{R}$ and $\lambda>0, \delta>0$, we have

$$
\mathbf{P}\left(\left|\frac{H_{n}}{n}(\omega, \eta)-\xi\right| \leq \delta\right)=\mathbf{P}\left(\mathrm{e}^{-\lambda n\left|\frac{H_{n}}{n}(\omega, \eta)-\xi\right|} \geq \mathrm{e}^{-\lambda n \delta}\right) \leq \mathrm{e}^{\lambda n \delta} \mathbf{P}\left(\mathrm{e}^{-\lambda\left|H_{n}-n \xi\right|}\right)
$$

Therefore,

$$
\begin{gathered}
\limsup \frac{1}{n} \log \nu_{n}([\xi-\delta, \xi+\delta]) \leq \lambda \delta-I^{(\lambda)}(\xi) \\
\limsup _{\delta \rightarrow 0} \lim \sup \frac{1}{n} \log \nu_{n}([\xi-\delta, \xi+\delta]) \leq-I^{(\lambda)}(\xi)
\end{gathered}
$$

and we obtain by letting $\lambda \rightarrow+\infty$,

$$
\limsup _{\delta \rightarrow 0} \lim \sup \frac{1}{n} \log \nu_{n}([\xi-\delta, \xi+\delta]) \leq-I(\xi) .
$$


Given $\xi \in \mathbb{R}$ such that $I(\xi)<+\infty$, and $\delta>0$, we have for $\lambda>0$,

$$
\mathbf{P}\left(\left|\frac{H_{n}}{n}-\xi\right|<\delta\right) \geq \mathbf{P}\left(\mathrm{e}^{-\lambda\left|H_{n}-n \xi\right|}\right)-\mathrm{e}^{-\lambda \delta n}
$$

Hence, if we choose $\lambda>0$ large enough such that $\lambda \delta>I(\xi) \geq I^{(\lambda)}(\xi)$, we obtain

$$
\liminf _{n \rightarrow+\infty} \frac{1}{n} \log \nu_{n}((\xi-\delta, \xi+\delta)) \geq-I^{(\lambda)}(\xi) \geq-I(\xi)
$$

and therefore

$$
\liminf _{\delta \rightarrow 0} \liminf _{n \rightarrow+\infty} \frac{1}{n} \log \nu_{n}((\xi-\delta, \xi+\delta)) \geq-I(\xi) .
$$

\section{ApPEndix A}

Exponential tightness plays the same role in Large Deviations theory as tightness in weak convergence theory; in particular it implies that the Large Deviations Property holds along a subsequence with a good rate function (see Thm. 3.7 of Feng and Kurtz [11], or Lem. 4.1.23 of Dembo and Zeitouni [10]). Therefore, once exponential tightness is established, we only need to identify the rate function: the Weak Large Deviations Property implies the Large Deviations Property with a good rate function (see Dembo and Zeitouni [10], Lem. 1.2.18). Our strategy of proof is then clear. First we establish exponential tightness, by applying the following lemma to the probability $\nu_{n}$ and the Lyapunov function $x \rightarrow|x|$, then we prove a Weak Large Deviations Property by checking that the limits (2.2) and (2.3) hold.

Lemma A.1. Let $\left(\mu_{n}\right)_{n \in \mathbb{N}}$ be a sequence of probability measures on a Polish space $X$. Assume that there exists $a$ (Lyapunov) function $F: X \rightarrow \mathbb{R}_{+}$such that the level sets $\{F \leq C\}_{C>0}$ are compacts, and

$$
\limsup _{n \rightarrow+\infty} \frac{1}{n} \log \left(\int \mathrm{e}^{n F(x)} \mathrm{d} \mu_{n}(x)\right)<+\infty .
$$

The $\left(\mu_{n}\right)_{n \in \mathbb{N}}$ is exponentially tight, i.e. for each $A>0$, there exists a compact $K_{A}$ such that:

$$
\limsup _{n \rightarrow+\infty} \log \mu_{n}\left(K_{A}^{C}\right) \leq-A \text {. }
$$

Proof. Let $M=\lim \sup _{n \rightarrow+\infty} \frac{1}{n} \log \left(\int \mathrm{e}^{n F(x)} \mathrm{d} \mu_{n}(x)\right)$. There exists $n_{0}$ such that for $n \geq n_{0}$,

$$
\frac{1}{n} \log \left(\int \mathrm{e}^{n F(x)} \mathrm{d} \mu_{n}(x)\right) \leq 2 M .
$$

Thanks to Markov inequality, for $C>0$ and $n \geq n_{0}$,

$$
\mu_{n}(F>C)=\mu_{n}\left(\mathrm{e}^{n F}>\mathrm{e}^{n C}\right) \leq \mathrm{e}^{-n C} \int \mathrm{e}^{n f} \mathrm{~d} \mu_{n} \leq \mathrm{e}^{-n(C-2 M)} .
$$

Hence, if $C>2 M+A$, then for the compact set $K_{A}=\{F \leq C\}$, and $n \geq n_{0}$,

$$
\frac{1}{n} \log \mu_{n}\left(K_{A}^{C}\right) \leq-(C-2 M)<-A .
$$

In Step 2 of the proof of the main theorem, we apply the following lemma to the family of functions $u_{n}=-v_{n}^{(\lambda)}$. 
Lemma A.2. Assume that the non negative functions $u_{n}: \mathbb{R} \rightarrow \mathbb{R}_{+}$are Lipschitz with the same constant $C>0$, that is

Assume furthermore the subadditivity:

$$
\forall n, x, y, \quad\left|u_{n}(x)-u_{n}(y)\right| \leq C|x-y| .
$$

$$
\forall x, y, n, m, \quad u_{n+m}(x+y) \leq u_{n}(x)+u_{m}(y) .
$$

Then there exists a non negative function $I: \mathbb{R} \rightarrow \mathbb{R}_{+}$, Lipschitz with constant $C$, that satisfies:

(i) if $\frac{a_{n}}{n} \rightarrow x$, then $\frac{1}{n} u_{n}\left(a_{n}\right) \rightarrow I(x)$.

(ii) $I$ is convex.

Proof. For fixed $x \in \mathbb{R}$, the sequence $z_{n}=u_{n}(n x)$ is subadditive and non negative:

$$
z_{n+m} \leq z_{n}+z_{m} .
$$

Therefore, by the standard subadditive theorem for sequences of real numbers, we can consider the limit

$$
I(x)=\inf _{n \geq 1} \frac{1}{n} z_{n}=\lim _{n \rightarrow+\infty} \frac{1}{n} z_{n}=\lim _{n \rightarrow+\infty} \frac{1}{n} u_{n}(n x) .
$$

If we take limits in the inequality

we obtain $|I(x)-I(y)| \leq C|x-y|$.

$$
\left|\frac{1}{n} u_{n}(n x)-\frac{1}{n} u_{n}(n y)\right| \leq C|x-y|
$$

(i) Assume $\frac{a_{n}}{n} \rightarrow x$, then

Hence, $\frac{1}{n} u_{n}\left(a_{n}\right) \rightarrow I(x)$.

$$
\left|\frac{1}{n} u_{n}(n x)-\frac{1}{n} u_{n}\left(a_{n}\right)\right| \leq C\left|x-\frac{a_{n}}{n}\right| \rightarrow 0 .
$$

(ii) We have, $\lfloor y\rfloor$ denoting the integer part of the real number $y$, for any $x, y$ and $0<t<1$,

$$
u_{\lfloor t n\rfloor+\lfloor(1-t) n\rfloor}(n t x+n(1-t) y) \leq u_{\lfloor t n\rfloor}(n t x)+u_{\lfloor(1-t) n\rfloor}(n(1-t) y) .
$$

Since $\frac{1}{n}(\lfloor t n\rfloor+\lfloor(1-t) n\rfloor) \rightarrow 1$, we have by (i)

$$
\frac{1}{n} u_{\lfloor t n\rfloor+\lfloor(1-t) n\rfloor}(n t x+n(1-t) y) \rightarrow I(t x+(1-t) y) .
$$

Furthermore, since $\frac{1}{n}\lfloor t n\rfloor \rightarrow t$, we have by (i),

$$
\frac{1}{n} u_{\lfloor t n\rfloor}(n t x) \rightarrow t I(x)
$$

and similarly,

$$
\frac{1}{n} u_{\lfloor(1-t) n\rfloor}(n(1-t) y) \rightarrow(1-t) I(y)
$$

Combining these limits with the preceding inequality yields,

$$
I(t x+(1-t) y) \leq t I(x)+(1-t) I(y)
$$

that is $I$ is convex.

Acknowledgements. The author wants to thank an anonymous referee for suggesting useful improvements to the original manuscript. 


\section{REFERENCES}

[1] K.S. Alexander, Approximation of subadditive functions and convergence rates in limiting-shape results. Ann. Probab. 25 (1997) 30-55. MR MR1428498.

[2] C. Ané, S. Blachère, D. Chafaï, P. Fougères, I. Gentil, F. Malrieu, C. Roberto and G. Scheffer, Sur les inégalités de Sobolev logarithmiques, Panoramas et Synthèses [Panoramas and Syntheses], volume 10. Société Mathématique de France, Paris (2000). With a preface by Dominique Bakry and Michel Ledoux. MR MR1845806.

[3] E. Bolthausen, A note on the diffusion of directed polymers in a random environment. Commun. Math. Phys. 123 (1989) 529-534.

[4] P. Carmona and Y. Hu, On the partition function of a directed polymer in a Gaussian random environment, Probab. Theory Relat. Fields 124 (2002) 431-457. MR MR1939654.

[5] P. Carmona and Y. Hu, Fluctuation exponents and large deviations for directed polymers in a random environment. Stoch. Process. Appl. 112 (2004) 285-308.

[6] P. Carmona and Y. Hu, Strong disorder implies strong localization for directed polymers in a random environment. ALEA 2 (2006) 217-229.

[7] F. Comets and N. Yoshida, Directed polymers in random environment are diffusive at weak disorder. Ann. Probab. 34 (2006) $1746-1770$.

[8] F. Comets and V. Vargas, Majorizing multiplicative cascades for directed polymers in random media. ALEA Lat. Am. J. Probab. Math. Stat. 2 (2006) 267-277 (electronic). MR MR2249671.

[9] F. Comets, S. Popov and M. Vachkovskaia, The number of open paths in an oriented $\rho$-percolation model. J. Stat. Phys. 131 (2008) 357-379. MR MR2386584.

[10] A. Dembo and O. Zeitouni, Large deviations techniques and applications. Second edition. Volume 38 of Applications of Mathematics (New York). Springer-Verlag, New York (1998). MR MR1619036.

[11] J. Feng and T.G. Kurtz, Large deviations for stochastic processes, in Mathematical Surveys and Monographs, volume 131. American Mathematical Society, Providence, RI (2006). MR MR2260560.

[12] H. Kesten and V. Sidoravivius, A problem in last-passage percolation, preprint (2007), http://arxiv.org/abs/0706.3626.

[13] S.R.S. Varadhan, Large deviations for random walks in a random environment. Commun. Pure Appl. Math. 56 (2003) 12221245. Dedicated to the memory of Jürgen K. Moser. MR MR1989232. 